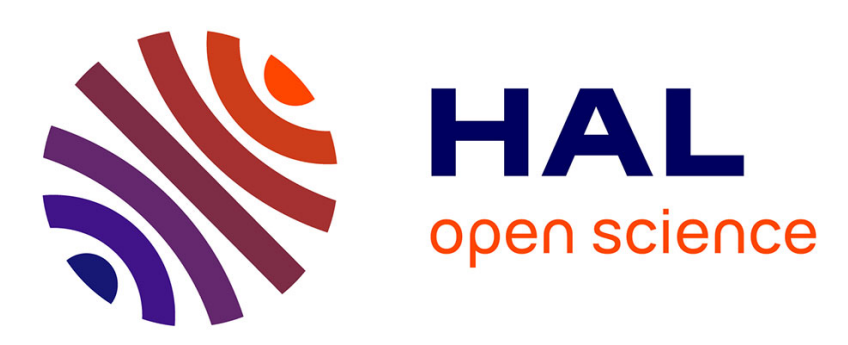

\title{
Direct Trajectory Interpolation on the Surface using an Open CNC
}

\author{
Xavier Beudaert, Sylvain Lavernhe, Christophe Tournier
}

\section{To cite this version:}

Xavier Beudaert, Sylvain Lavernhe, Christophe Tournier. Direct Trajectory Interpolation on the Surface using an Open CNC. International Journal of Advanced Manufacturing Technology, 2014, 75, pp.535-546. 10.1007/s00170-014-6134-7 . hal-01055090

\section{HAL Id: hal-01055090 https://hal.science/hal-01055090}

Submitted on 11 Aug 2014

HAL is a multi-disciplinary open access archive for the deposit and dissemination of scientific research documents, whether they are published or not. The documents may come from teaching and research institutions in France or abroad, or from public or private research centers.
L'archive ouverte pluridisciplinaire HAL, est destinée au dépôt et à la diffusion de documents scientifiques de niveau recherche, publiés ou non, émanant des établissements d'enseignement et de recherche français ou étrangers, des laboratoires publics ou privés. 


\title{
Direct Trajectory Interpolation on the Surface using an Open CNC
}

\author{
Xavier Beudaert · Sylvain Lavernhe · Christophe Tournier
}

Received: date / Accepted: date

\begin{abstract}
Free-form surfaces are used for many industrial applications from aeronautical parts, to molds or biomedical implants. In the common machining process, CAM software generates approximated tool paths because of the limitation induced by the input tool path format of the industrial CNC. Then, during the tool path interpolation, marks on finished surfaces can appear induced by non smooth feedrate planning. Managing the geometry of the tool path as well as the kinematical parameters of the machine tool are two key factors for quality and productivity improvements. The aim of this paper is to present a unified method to compute the trajectory directly on the surface to be machined avoiding CAM approximations and producing a smoother trajectory. This paper proposes an interpolation of the trajectory based on the free form surface mathematical model while considering the kinematical limitations of a high speed milling machine (velocity, acceleration and jerk). The amelioration of the data exchange between CAD/CAM and CNC opens new ways to optimize the manufacturing process. The Direct Trajectory Interpolation on the Surface (DTIS) method allows to obtain both a higher productivity and a better surface quality. Machining experiments carried out with an Open CNC on a 5-axis high speed milling machine show the benefits of the proposed method compared to the classical strategies available with an industrial $\mathrm{CNC}$.
\end{abstract}

Keywords surface interpolation - 5-axis machining · feedrate planning $\cdot$ jerk limitation $\cdot$ open $\mathrm{CNC}$

X. Beudaert - S. Lavernhe - C. Tournier

LURPA, ENS Cachan, Univ Paris-Sud, F-94235 Cachan, France

Tel.: +33147402996

Fax: +331474022 20

E-mail: christophe.tournier@lurpa.ens-cachan.fr

\section{Introduction}

3 and 5-axis High Speed Milling machines are often used to generate free-form surfaces for mold and die, aeronautical or biomedical industries. Various sources of geometrical deviations on part coming from all the elements of the manufacturing process have been identified in the literature. These errors are for example linked to the Computer Aided Manufacturing (CAM) programs (tool path computation), to the CNC (interpolation, inverse kinematical transformation, axis follow-up), to the static, dynamic and thermal errors of the machine tool (machine geometry) or to process related errors (actual tool geometry, tool deflection, vibrations, chip formation, etc.).

As it is can be seen in [26], the tool path interpolation can be one of the main source of problem regarding the productivity and the quality of the free form surface machining process. 5-axis surface machining has several advantages compared to 3-axis surface machining such as increased material removal rate, tool length reduction, better surface finish, etc. However, 3 and 5-axis machining strategies encounter the same problems concerning the linear discretization of the tool path which reduces the performance of the machining process.

In the typical manufacturing process for free-form surface machining, the CAM program discretizes the continuous free-form curves in small segments. Indeed, the linear interpolation (G1) is the most commonly used format to describe the tool paths in current industrial CNCs. However, the G1 discretization brings several major drawbacks:

- The linear interpolation of a curve induces geometrical deviations. Even though no physical process is involved, a chord error is generated between the ideal tool path and the discretized one in CAM software by the use of a machining tolerance. These geometrical deviations can be reduced at the expense of the amount of data. 
- The amount of data required to describe a simple parametric curve can be huge and these data have to be treated in real time by the CNC. Moreover, small segments can induce a reduction of the feedrate because of the block processing time of the CNC. Hence, the geometrical deviations cannot be reduced to a negligible value with the linear interpolation while keeping high productivity.

- The segments which discretized the continuous curves present tangency discontinuities. So even if the profile to be machined is really smooth, the tool path geometry is only $\mathscr{G}^{0}$ continuous with the linear interpolation which leads to the following disadvantages.

- The maximum reachable feedrate along the machined profile is limited by the discontinuous geometry generated with the linear interpolation. The length and the angle between the segments can have an important effect on the feedrate; the relation between these parameters and the actual feedrate is not trivial.

- Finally, the G1 discretization is harmful for the surface finish. Indeed, if tool paths are synchronized, facets can appear on the machined surface. The use of desynchronization may also induce marks, called "skin effect", on the surface. Moreover, the feedrate variations caused by the discontinuities can also leave marks of the free-form surface.

Smart CNC algorithms have been developed to reduce these effects. However, the results presented in this paper should convince that for free-form surface machining applications, even compared to the advanced B-Spline interpolations available in the market, significant quality and productivity improvements can be obtained using a better representation and interpolation of the tool path.

In the literature, several propositions have been made to improve the manufacturing process of free-form surfaces. Three approaches can be distinguished: first, different techniques are implemented in the CNC to smooth the tool path. Second, improved CAM algorithms are proposed to generate better tool paths. Third, new tool path formats are proposed to avoid the CAM discretizations.

$\mathrm{CNC}$ tool path smoothing techniques have been presented in the literature to round the discontinuities created by the linear interpolation. In order to obtain a $\mathscr{G}^{2}$ continuous tool path, a $5^{\text {th }}$ order polynomial curve is used to round the corners in [6]. A cubic B-Spline with eight control points is used in [21] to round 3-axis G1 corners whereas a cubic BSpline with five control points is used in [3] to round 3 and 5 -axis discontinuities. CNC builders also developed efficient algorithms to smooth the tool path geometry. Siemens 840D CNCs offer corner rounding methods [28] or online Spline interpolation with compressor functions (see CompCad in [23]). This kind of online Spline interpolation is detailed in a patent owned by Fanuc [20]. These tool path smoothing method combined with look ahead strategies reduce the ef- fects of the G1 discontinuities and thus improve the productivity. However, on top of the CAM tolerance, a CNC tolerance is required which induces again geometrical deviations between the tool path and the surface to be machined. Moreover, the performances of these complex algorithms are limited by the real-time constraint. Finally, the improvements that can be achieved within the CNC systems are limited because of the lack of information about the machined surface.

Ameliorations of CAM algorithms have been proposed to improve the tool path sent to the CNC. A circular arc or biarc curve interpolation able to reduce the amount of data and to increase the continuity of the tool path is presented in [27]. $\mathscr{G}^{1}$ continuity is also obtained in [4] with linear (G1) and circular (G02/G03) interpolation. Circular as well as polynomial interpolation has been studied in [24]. Surface quality improvements have been observed with the improvement of the continuity of the tool path. However, circular interpolation techniques are not able to represent a free-form curve efficiently. Thus, Spline or polynomial curves are more suited for free-form machining. An algorithm to generate a cubic B-Spline tool path and thus to improve the surface quality while reducing the amount of data is proposed in [14]. In [15], a polynomial surface format is presented and native B-Spline curves are sent to the CNC. In [29], an arc length parameterized NURBS CL path with path errors and parameterization errors globally bounded is presented. Advanced CAM and CNC systems such as Catia v5 and Siemens $840 \mathrm{D}$ can allow a native B-Spline interpolation which means that the $\mathrm{NC}$ code sent to the numerical controller contains the mathematical expression of the B-Spline curves representing the tool path. The native B-Spline interpolation is the best solution currently available to get a smooth tool path with high productivity and improved surface finish.

No matter which previously cited strategy is used, the CAM tool path sent to the $\mathrm{CNC}$ is an approximation of the exact tool path. The result is that the CAM tool path is oscillating around the exact tool path which reduces the smoothness and induces geometrical errors, Fig. 1. The improvements that can be achieved by the CAM programs are limited because of the lack of possibilities available in the $\mathrm{NC}$ code to specify the tool path geometry.

The third option is to use the exact tool path geometry instead of an approximation. This option should obviously be preferred but as the NC code interpreted by industrial CNCs is really limited in terms of geometrical entities, this proposition cannot be implemented on current industrial machines. The work carried out around the Step-NC standard [9] intends to enhance the communication possibilities between CAM and CNC. This new standard will allow to specify a tool path with a complex mathematical expression. However, for the moment Step-NC compliant CNC exist only in laboratories [22] so the new tool path description 

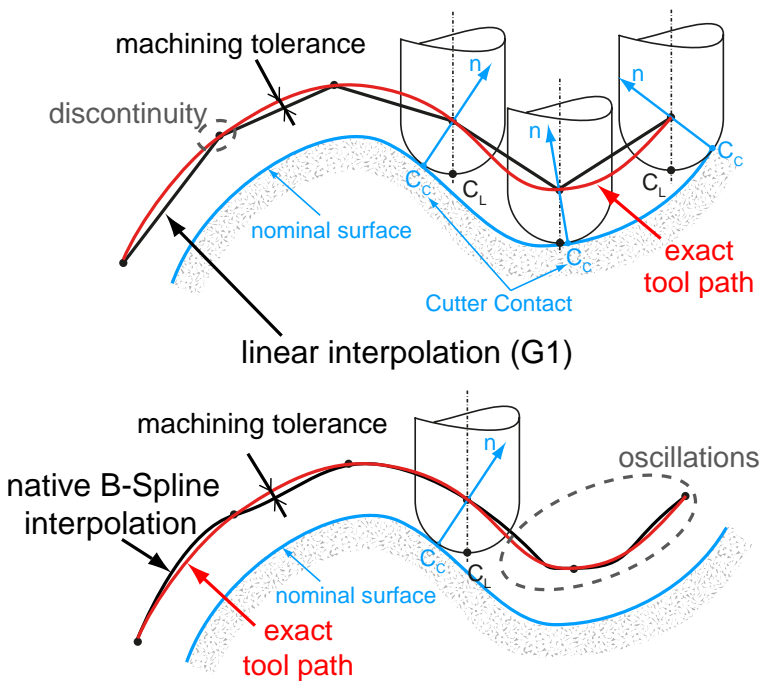

Fig. 1 Linear (G1) and native B-Spline interpolation

proposed in this paper is linked to the development of an Open CNC.

Exact interpolation on the surface has been proposed first by Koren in 1995 [12]. A new G code instruction is proposed to define the surface to be machined and a real-time interpolator computes the axis setpoints based on this exact geometry. This work is extended in [17], however in both articles a second order Taylor expansion is used to obtain a constant feedrate. These articles emphasize the geometrical benefits of the surface interpolation but the machining time reduction is not demonstrated. Then several papers focused their attention on the difference between the Cutter Location (CL) feedrate and the Cutter Contact (CC) feedrate $[18,25,5]$. However this difference does not seem to have an important impact for milling applications.

Experimental validations of surface interpolation are carried out on 3-axis machines in [25, 5, 19], but the physical constraints of high speed machining are not considered, especially the physical limitations on velocity, acceleration and jerk of the axes which limit the actual feedrate [2]. Recently, a 5-axis surface machining controller has been proposed by Liang and $\mathrm{Li}$ in [16]. Instead of defining new $\mathrm{G}$ code instruction such as in $[12,17,18,19,8]$, the Step-NC standard is used to define the tool path. A NURBS freeform surface is machined in 5-axis with an in-house StepNC compliant machine [16]. This work is really promising but as in all the previously published papers, the kinematical limitations on the axes are not considered.

To prove the benefits carried out by the exact surface interpolation compared to G1 discretization, it is important to include kinematical constraints in the feedrate interpolation. To the best of our knowledge, no previously published paper addressed the problem of exact surface interpolation with kinematical limitations. Moreover, the experimental valida- tion requires a high speed milling machine equipped with an Open CNC to overcome the limitations caused by the NC code format.

The aim of this paper is to interpolate the trajectory directly on the surface to be machined bringing two new contributions. First, the DTIS (Direct Trajectory Interpolation on the Surface) method computes the trajectory exactly on the surface while considering kinematical constraints (velocity, acceleration and jerk) of each axis. Second, a 5-axis high speed milling machine equipped with an Open CNC and a Siemens 840D CNC allows to compare the new strategy with the best standards available with an industrial CNC. Thus, a fair comparison can be made concerning machining time reduction and surface quality improvements.

The rest of the paper is organized as follows: the DTIS method is presented in Section 2. Section 3 presents the development carried out to build an Open CNC able to use this new interpolation method. In the last section, a surface is machined in 5-axis to compare the classical (G1 and BSpline) strategies to the proposed DTIS method.

\section{Direct Trajectory Interpolation on the Surface}

The DTIS method aims at computing axis setpoints which are exactly on the surface which is machined thus avoiding the geometrical errors classically introduced by the CAM approximations. First, the exact definition of the tool path is given as well as the method to compute it. Then, the trajectory interpolation with kinematical constraints is performed.

A distinction is made between the words "tool path" and "trajectory". For the rest of the paper, the word "tool path" is used to refer to the geometry of the trajectory whereas the word "trajectory" refers both to the geometry and to the temporal evolution along this geometry. NC code contains the tool path and the programmed feedrate, the CNC computes the trajectory with an actual feedrate which is often lower than the programmed feedrate depending on the kinematical characteristics of the machine.

\subsection{Definition of the tool path}

As it has been said in the introduction, the NC code which is currently used does not allow to define a tool path based on a mathematical expression. With the Step-NC format designed to replace the old $\mathrm{G}$ code, the tool path format is not limited anymore and it will be possible to define the tool path using high level information.

The free-form surface is expressed as a B-Spline surface using the Step format. The Step-NC ISO14649-11 standard [10] already contains the entities to define the machining strategies as well as the tool parameters. With the proposed 
DTIS method, the tool path is defined by the following high level entities:

- the surface $S(u, v)$ to be machined

- the machining strategy ("uv_strategy", "plane_cl_strategy", "plane_cc_strategy", "leading_ line_ strategy")

- the tool orientation

- the cutting tool geometrical and technical parameters

It is also important to specify the tool path when the tool is not in contact with the surface. The specific management of the tool path for approach and retract movements is also defined in [10] with the "Approach_ retract_ strategy" abstract supertype.

With this definition of the tool path, the NC program contains the information about the surface which is machined. The amount of data is reduced but the quality is improved, nevertheless new algorithms have to be integrated in the CNC. The amelioration of the data connection between CAD/CAM and $\mathrm{CNC}$ will open new ways to optimize the manufacturing process.

\subsection{Link between the CC and CL points}

The relation between the Cutter Contact (CC) point and the Cutter Location (CL) point depends on the geometry of the tool and on its orientation. To be as general as possible, a bull nose tool with a 5-axis orientation is used. The expressions for ball end or flat end mills are particular cases of the following formulas.

Using classical definition of the tool orientation, the tilt and yaw angles $\left(\theta_{t}\right.$ and $\left.\theta_{n}\right)$ are defined in the $(\mathbf{f}, \mathbf{n}, \mathbf{t})$ basis (feed direction, surface normal, transverse direction) by the rotations $\operatorname{Rot}_{\theta_{t}}$ and $\operatorname{Rot}_{\theta_{n}}$ respectively around the vectors $\mathbf{t}$ and $\mathbf{n}$.

$\operatorname{Rot}_{\theta_{t}}=\left[\begin{array}{ccc}\cos \theta_{t} & \sin \theta_{t} & 0 \\ -\sin \theta_{t} & \cos \theta_{t} & 0 \\ 0 & 0 & 1\end{array}\right]$

$\operatorname{Rot}_{\theta_{n}}=\left[\begin{array}{ccc}\cos \theta_{n} & 0 & \sin \theta_{n} \\ 0 & 1 & 0 \\ -\sin \theta_{n} & 0 & \cos \theta_{n}\end{array}\right]$

Thus, the combination of the two rotations makes the transformation between the normal vector $\mathbf{n}$ and the tool orientation vector $\mathbf{u}$.

$\mathbf{u}=\operatorname{Rot}_{\theta_{n}} \cdot \operatorname{Rot}_{\theta_{t}} \cdot \mathbf{n}$

Once the tool orientation is obtained, the link between the $\mathrm{CC}$ and the CL points is given in Eq. 4 with $\mathrm{R}$ the radius of the tool, and $r$ the small radius of the bull nose cutter.
When the $\mathrm{CC}$ point is known this expression gives an explicit formula to get the CL point. However, when the CL point is known, this expression has to be solved recursively to find the $\mathrm{CC}$ point and the corresponding normal vector $\mathbf{n}$

$\mathbf{C L}=\mathbf{C C}+r \mathbf{n}+(R-r) \mathbf{v}-r \mathbf{u}$ with $\mathbf{v}=\frac{\mathbf{u} \wedge \mathbf{n}}{\|\mathbf{u} \wedge \mathbf{n}\|} \wedge \mathbf{u}$

\subsection{Tool path description}

Using the high level definition of the tool path, the exact position of the CL point can be computed as well as the tool orientation. The general implicit problem for tool path computation can be written as follows:

$\{$ Exact position of the CC points on the surface $S(u, v)$

Compliance with the machining strategy

For an iso-parametric strategy ("uv_ strategy"), this system is solved explicitly. The location of the $\mathrm{CC}$ points is given by:

$C C(v)=S(u=c s t, v)$

and the CL point is obtained directly with Eq. 4.

The problem is more complex with a "plane _ cl_ strategy" than with a "plane_cc_strategy". Indeed, with a "plane cc s strategy" (plane denoted $\Pi_{C C}$ ), the position of the CC point is defined implicitly by Eq. 7 and the Eq. 4 is used to compute the CL point directly:

$$
\left\{\begin{array}{l}
C C \in S(u, v) \\
C C \in \Pi_{C C}
\end{array}\right.
$$

For a "plane_ cl_strategy", Eq. 8 has to be solved. Implicit expressions have to be used both for the exact position of the CC point on the surface and for the link between the $\mathrm{CC}$ and CL points (Eq. 3-4).

$$
\left\{\begin{array}{l}
C C \in S(u, v) \\
C L \in \Pi_{C L}
\end{array}\right.
$$

It is now possible to declare the exact tool path according to the machining strategy. However, up to now only the geometry of the trajectory has been defined. Within the high speed machining context, it is important to compute a feedrate profile along the tool path while considering the kinematical constraints of the axes. The computation of the geometry as well as of the feedrate will now be performed in a unified algorithm. 


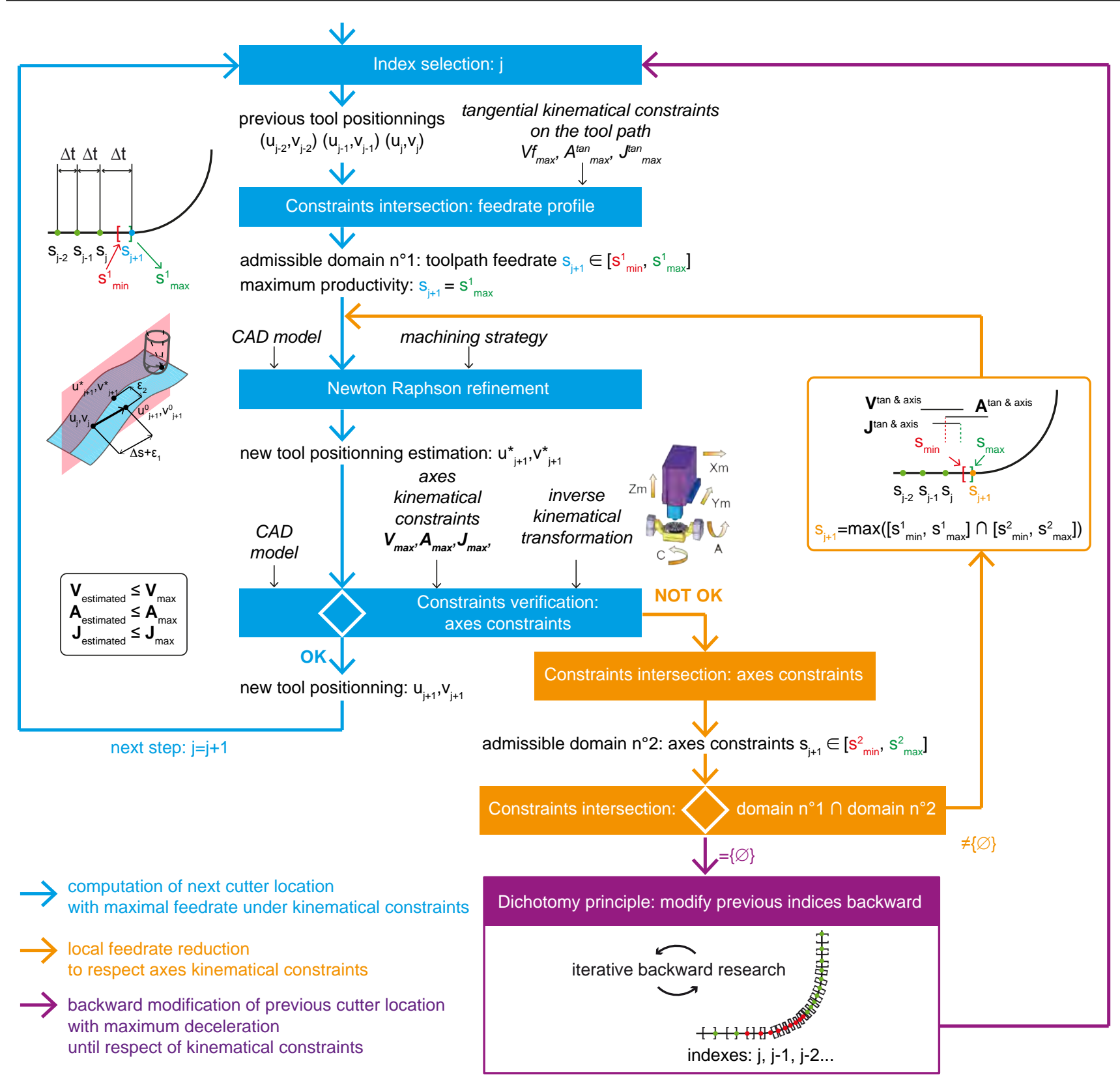

Fig. 2 DTIS method for trajectory interpolation

\subsection{DTIS principle}

Each drive has its own kinematical limits in terms of velocity, acceleration and jerk. The role of the feedrate interpolator is to create the motion law respecting all the kinematical constraints along the tool path and especially the axis jerk. The axis jerk often limits the actual feedrate and it is an important parameter to avoid excessive vibrations in high speed machining. This section will show how the DTIS method can merge, in a unique scheme, tool path computation and feedrate interpolation. The feedrate interpolation is based on the VPOp algorithm (Velocity Profile Optimization) previously developed [2].
The DTIS method is composed of three main loops, Fig. 2:

- The main loop (in blue) is the trajectory interpolation: it consists in computing new tool positioning from previous ones while verifying kinematical constraints.

- When axis kinematical constraints are not satisfied by the initial tool positioning, the second loop (in orange) modify the local feedrate by estimating an admissible domain along the trajectory.

- The third loop (in purple) deals with situations where no admissible solution can be found (empty interval). Typically, on portions of tool paths with high curvature, it is necessary to reduce feedrate before entering the curve. If the feedrate is too high at the entrance of a high curva- 
ture area, a backward scan is performed to find the point from which it is necessary to decelerate.

The main contribution of the proposed DTIS method is to use the exact tool path definition and to respect the kinematical constraints.

\subsubsection{Tool path interpolation}

The tool path interpolation is performed directly into the parametric space of the surface to cancel errors from tool positioning. Moreover with DTIS method, since there is no more association of linear or polynomial format, all computed setpoints are by definition exactly on the surface without chord error.

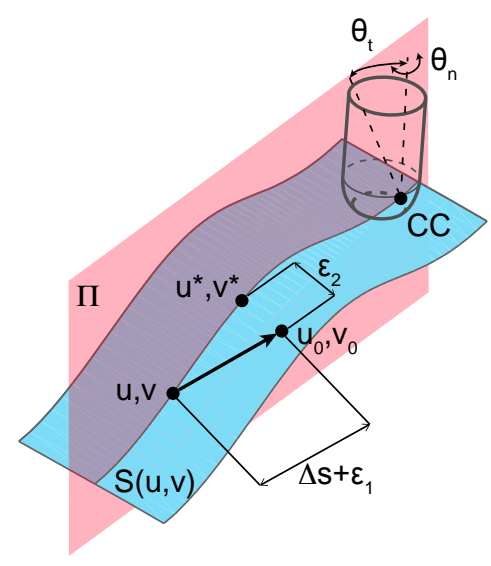

Fig. 3 Exact tool path computation

To solve the implicit problem of tool path computation with "plane_ cl_strategy", a Newton-Raphson method is illustrated in Fig. 3. Starting from the point $(u, v)$ of the machined surface, the first guest $\left(u_{0}, v_{0}\right)$ is computed assuming an evolution with maximum productivity (distance $\Delta s$ ).Then, the errors $\varepsilon_{1}$ and $\varepsilon_{2}$ are minimized to compute the next point $\left(u^{*}, v^{*}\right)$ on the tool path according to the machining strategy. As the machined surface is usually really smooth, the first guess is really close to the exact value, only one or two iterations can be necessary to set the tool positioning with an error under $0.001 \mu \mathrm{m}$. Such a threshold is negligible for high speed machining applications. Thus with this iterative method, the tool path can be computed exactly on the surface.

\subsubsection{Kinematical constraints from the tool path}

The interpolation of the tool path is based on the estimation of the feedrate profile. Indeed, from the previous tool positionings $\left(u_{j-2}, v_{j-2}\right),\left(u_{j-1}, v_{j-1}\right),\left(u_{j}, v_{j}\right)$ and their positions on the trajectory $\left(s_{j-2}, s_{j-1}, s_{j}\right)$ the feedrate profile is defined with the tangential kinematical constraints
$V f_{\text {max }}, A_{\text {max }}^{\tan }, J_{\text {max }}^{\text {tan }}$. Considering that maximal and minimal constraints are symmetrical and the fixed temporal step size $\Delta t$ of the interpolator cycle time, it is possible to determine the admissible domain of the tool path for $s_{j+1}$. Hence, the corresponding spatial step $(\Delta s)$ on the trajectory is used during the interpolation stage to set the new tool positioning $\left(u^{*}, v^{*}\right)$ :

$\Delta s=s_{j+1}-s_{j}$

To maximize the efficiency of machining and the productivity, the farthest point is chosen by default $\left(s_{j+1}=s_{\text {max }}^{1}\right)$.

\subsubsection{Kinematical constraints from the machine tool axes}

Once the interpolation stage is performed, kinematical solicitations on each axis resulting from the previous tool positionings are compared to the axis constraints. Kinematical solicitations are evaluated exactly since tool positionings are previously evaluated using the CAD model and the inverse kinematical transformation of the machine tool are known. One can find more details on the equations of kinematical characteristics in [2].

If constraints are satisfied, the current tool positioning is validated and the algorithm goes to next iteration. If not, the first stage is to locally modify the current tool positioning to respect axis constraints (orange loop on Fig. 2). On the first tool positioning, axis constraints are used to evaluate the admissible domain $\left(\left[s_{\text {min }}^{2}, s_{\text {max }}^{2}\right]\right)$. This domain can then be intersected with the domain $\mathrm{n}^{\mathrm{O}} 1$ to find a new tool position $s_{j+1}$ and continue the main loop by the Newton-Raphson refinement.

Sometimes, when feedrate needs to be decreased, the intersection of admissible domains $n^{\circ} 1$ and $n^{0} 2$ is empty $(=\{\varnothing\})$, Eq. 10.

$s_{j+1}=\min \left(\left[s_{\min }^{1}, s_{\max }^{1}\right] \cap\left[s_{\min }^{2}, s_{\max }^{2}\right]\right)$

Hence, within the purple loop, previous tool positionings are modified. An iterative backward research allows to find a minimal sequence of setpoints to decelerate and reach the admissible kinematical level to respect constraints. To do so, the lower bound of the admissible domains intersection is selected: criterium of maximum feedrate increase is switched to maximum feedrate decrease. This method goes then backward to modify previous computed points until tool positioning of index $j$ is solved. The efficiency of the iterative research can be notably improved by using a dichotomy principle and look ahead anticipation [2].

To conclude, the Direct Trajectory Interpolation on the Surface allows to compute axis setpoints which describe exactly the geometry of the surface. Hence, the geometrical 
deviations and discontinuities introduced by the CAM programs are avoided and the tool path can be more accurate and smoother. These improvements come from a stronger communication between CAM and CNC with the Step-NC standard able to transfer better tool path definitions.

\section{PREMIUM-OpenCNC}

As it has been said in the introduction, the exact surface interpolation cannot be performed using an industrial CNC. Thus, it is necessary to develop an Open CNC in order to overcome the limitations of the NC code and to control directly the movements of the machine. This section will present the development carried out to build an Open CNC system connected to an industrial 5-axis high speed machine. An interesting advantage of the hybrid solution used is that the same machine can be controlled with a Siemens 840D CNC and with an Open CNC, hence fair comparisons can be made to demonstrate the benefits of the Direct Trajectory Interpolation on the Surface.

\subsection{Hybrid integration of the Open CNC in the Siemens} 840D CNC

The PREMIUM-OpenCNC has been developed to control the motion of a 5-axis Mikron UCP710 high speed milling machine without using the Siemens 840D CNC originally installed on the machine. Indeed, although the 840D CNC allows the access to numerous variables, the PLC, synchronous functions or specific libraries, the choice was done to develop our own CNC to highlight improvements on the trajectory definition and interpolation stages, without any limitation to the Siemens kernel. Another possibility would be to use the LinuxCNC [1] which represents an interesting attempt to offer an open source CNC. However it does not yet provide satisfactory algorithms for jerk limited feedrate interpolation in 5-axis machining which is crucial for high speed machining.

To reduce the development efforts, the Siemens $840 \mathrm{D}$ is not totally disconnected. Indeed, the Siemens CNC is still used for auxiliary tasks (cooling system, safety, PLC behavior...) and to control the spindle. However, it does not have any connection with the displacement axes which are completely controlled by the PREMIUM-OpenCNC.

The second step to be able to move the machine using the PREMIUM-OpenCNC is to send the setpoints to the motor drive. In the Siemens Powerline CNC, the 611D motor drives are connected to the CNC with the drive bus, Fig. 5. A Siemens proprietary Profibus-DP protocol is used for the communication. As it is difficult to use this communication bus to control the drives, the 611D Digital control board are

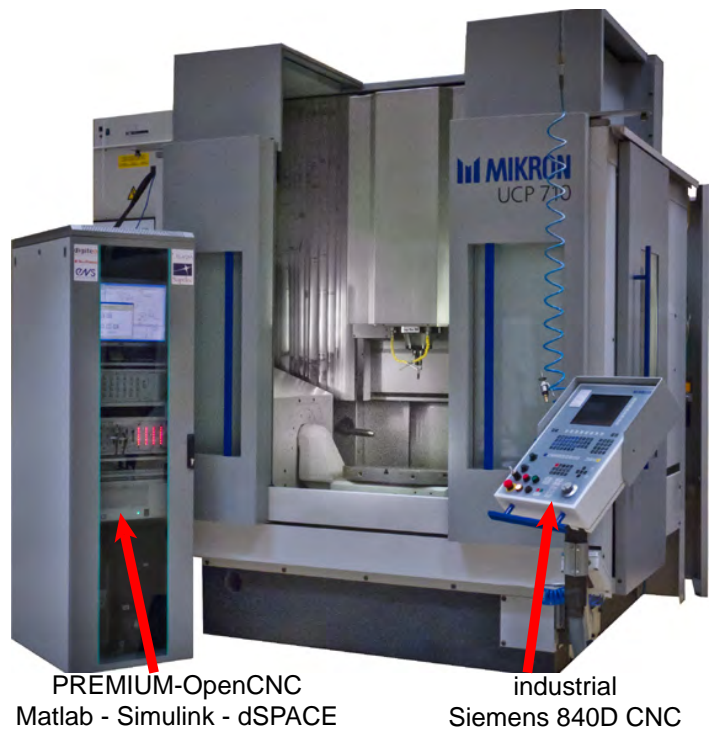

Fig. 4 5-axis high speed milling machine with the PREMIUMOpenCNC and the Siemens 840D CNC

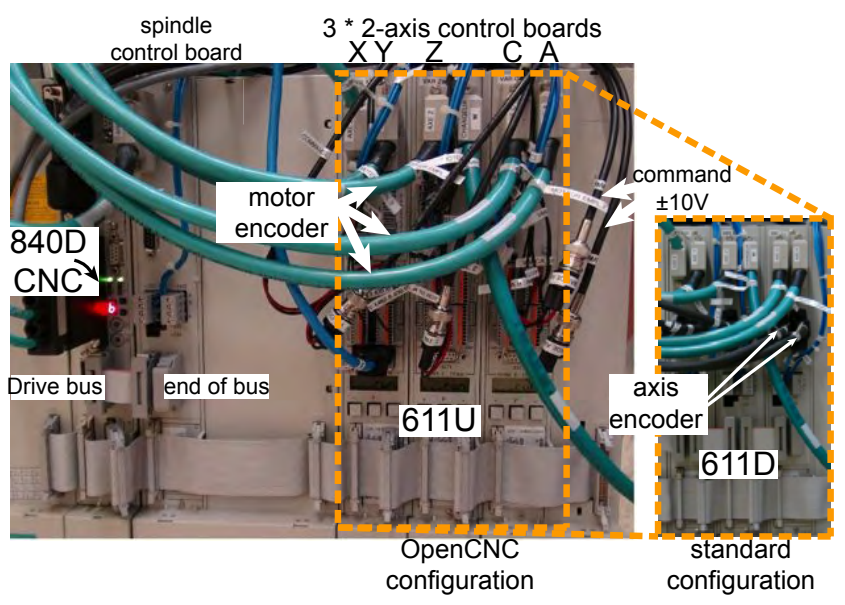

Fig. 5 Open $\mathrm{CNC}$ and standard configuration of the control boards

replaced by $611 \mathrm{U}$ universal control board which can easily be controlled by a $\pm 10 \mathrm{~V}$ command voltage. The original and modified configuration can be seen in Fig. 5. The time required to switch from the standard configuration to the PREMIUM-OpenCNC is around $1 \mathrm{~h} 30$. That means that it is easily possible to machine the same workpiece using the two configurations to compare the surface finish as it will be shown in the application.

The machine is controlled with a typical cascade structure with current, velocity and position control loops. The motor control boards perform the current and velocity loops; that is why the motor encoders are plugged on them. In the $611 \mathrm{D}$ configuration, the axis encoders are also plugged to the motor drive but this information is sent back to the Siemens 840D CNC which performs the position control loop. In the Open CNC configuration, this information is not sent anymore to the Siemens $840 \mathrm{D} \mathrm{CNC}$ and it is directly 
used by the real-time computer used for the PREMIUMOpenCNC controller. Finally, to be able to control the machine one just need to send to command voltage which corresponds to the command velocity of the motor.

Once this technical implementation is performed, the trajectory can be sent directly to the motor control boards avoiding all the unknown treatments realized by the industrial CNC. To move the axes, a real-time controller has been developed in order to close the position loop and to generate the velocity commands.

\subsection{Real-time control of the PREMIUM-OpenCNC}

The PREMIUM-OpenCNC is based on a modular dSPACE real-time system. An incremental encoder interface is used to read the position directly on the encoders of the drives and a high resolution Digital/Analog card converts the setpoints in a $\pm 10 \mathrm{~V}$ signal. The position setpoints are preprocessed with Matlab using a standard computer whereas a dSPACE DS1006 processor board is used to perform all the computations needed for the real-time control. The dSPACE environment is user friendly as a Simulink model can automatically be compiled for the real-time execution. Indeed, the dSPACE Real Time Interface provides all the Simulink blocks to connect the model to the physical input / output. Hence, all the development is made with Simulink and the user interface is realized with the software ControlDesk furnished by dSPACE. The cost of hardware and realtime solution could be estimated to $30 \mathrm{k} €$.

To obtain a low tracking error with high speed movements, it is necessary to implement a feedforward strategy on top of the feedback control. So a velocity feedforward is tuned according to the dynamic response of the velocity control loops of the axes. In 5-axis machining, a desynchronization of the axes can cause large contour errors on the workpiece. As the linear and the rotary axes have different response time, it is also necessary to introduce a dynamical adaptation of the setpoints. All these developments are out of the scope of this paper so they are not explained in details. Nevertheless, with this efficient control tuning, the contour error generated with the OpenCNC are acceptable for 5-axis high speed machining.

Finally, the 5-axis high speed Mikron machine can be used both with a standard Siemens 840D CNC and with an Open CNC where the setpoint generation is completely under control. This experimental platform is well suited for the implementation of the Direct Trajectory Interpolation on the Surface presented in this paper. This platform offers a unique opportunity to compare new scientific advances with the best industrial standards.

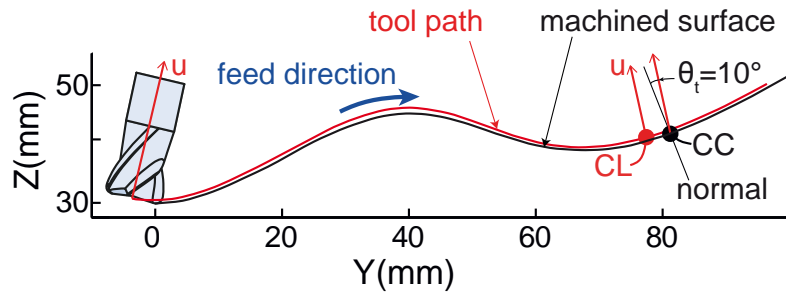

Fig. 6 5-axis tool path with $10 \mathrm{deg}$ tilt angle

Table 1 Machining strategies

\begin{tabular}{lllll}
\hline & Strategy & CNC & NC blocks & Time (s) \\
\hline 1 & G1 10 $10 m$ & 840D & 59 & 14.4 \\
2 & G1 1 $\mu m$ & 840D & 189 & 12.2 \\
3 & B-Spline 10 $\mu m$ & 840D & 85 & 8 \\
4 & B-Spline 1 $\mu \mathrm{m}$ & 840D & 226 & 9 \\
5 & DTIS & Open CNC & - & 3 \\
\hline
\end{tabular}

\section{Application}

The DTIS method presented in this paper is applied on a 5axis tool path, Fig. 6. An aluminum workpiece is machined with a bull nose cutter with 5 different strategies. The programmed feedrate $V f$ is $3 \mathrm{~m} / \mathrm{min}$. For each strategy, the tilt angle is fixed at $10 \mathrm{deg}$ with no yaw angle which produces a 5-axis tool path with a simultaneous movement of $\mathrm{Y}, \mathrm{Z}$ and $A$ axes along one path. The machined surface is chosen to emphasize the benefits of the DTIS method. However, any free-form surface machining operation can be improved with the proposed surface interpolation even in 3-axis machining. As the surface is ruled in the $\mathrm{X}$ direction, one pass in the YZ plane is repeated with a step over distance of $2 \mathrm{~mm}$ to machine the surface. The tool path lays in a plane, so it is easier to measure and to represent the effect of the tool path format on the feedrate. Moreover, the synchronization of the passes in the $\mathrm{X}$ direction will leave obvious marks on the machine surface as underlined further.

- The first two strategies use the most popular format which is the linear interpolation (G1) with two different CAM tolerances, $10 \mu \mathrm{m}$ and $1 \mu \mathrm{m}$ respectively. In the table 1 , the number of NC blocks used to describe one pass is collected as well as the machining time. With a higher CAM tolerance, the amount of data is reduced but it is important to notice that the machining time is increased. There is no direct connection between the machining time and the number of blocks, depending on the angles between the segments the feedrate variation is changing as pointed out in [2].

- The strategies 3 and 4 use the native B-Spline format generated with Catia V5 CAM software and interpreted directly by the Siemens 840D CNC. Here also two different CAM tolerances are tested. A tolerance of $10 \mu \mathrm{m}$ 
is standard for general purpose curve interpolation, with $1 \mu \mathrm{m}$ the chord error is really small for precise finishing operations. With the NURBS format that can be rarely found within the CAM-CNC community, this native BSpline interpolation is the best solution industrially available for free-form surface machining. Indeed, a continuous B-Spline tool path is created by the CAM software so the CNC does not have to run complex smoothing algorithms in real-time. As the tool path geometry is smoother than with a G1 tool path smoothed by the $\mathrm{CNC}$, the machining time is reduced and the surface finish is improved.

- The last portion of the workpiece is machined with the newly developed DTIS method. For this surface, the DTIS algorithm performs both tool positionings and feedrate interpolation in a synchronous manner, the PREMIUMOpenCNC replace the commercial CAM and CNC solutions. As there is no discretization of the tool path, the number of blocks is meaningless for this strategy because cutter locations are computed during the interpolation on a portion of the trajectory. The machining time is reduced by more than twice compared to the best industrial solution, i.e. the native B-Spline interpolation and by more than four times compared to the commonly used G1 format. This machining time reduction is explained by the better tool path smoothness obtained with the direct interpolation on the surface. The reason for this difference of smoothness between the DTIS tool path and the B-Spline tool path is quite clear. As the B-Spline tool paths are approximated, they are oscillating around the theoretical curve which is the smoothest tool path.

The measured feedrates are presented in Fig. 7. For the G1 $10 \mu \mathrm{m}$ strategy, it is clear that the feedrate have strong oscillations along the path. The discontinuities at the end of each NC block induce feedrate slowdowns. With the second strategy G1 $1 \mu \mathrm{m}$, the discontinuities are reduced so as the feedrate variations. For the B-Spline strategies, the tool path is smoother so the feedrate profile is also smoother. However, the programmed feedrate of $3 \mathrm{~m} / \mathrm{min}$ is never reached with the industrial strategies. The DTIS strategy allows to significantly increase the feedrate and even to reach the programmed feedrate at the end of the tool path. At the beginning, the programmed feedrate cannot be reached because of the acceleration and jerk limitations of the A axis which is highly excited.

The feedrate profiles presented in the previous figure are plotted on top of the tool path geometry in Fig. 8. As it is a 5-axis tool path, the analysis of the feedrate reduction is difficult because it is not directly linked to the curvature of the tool path. However, for the first strategy, the feedrate oscillations correspond to the programmed position and orientation of the G1 NC blocks. Around $Y=20 \mathrm{~mm}$, there is a long segment where the feedrate can be higher but as we will

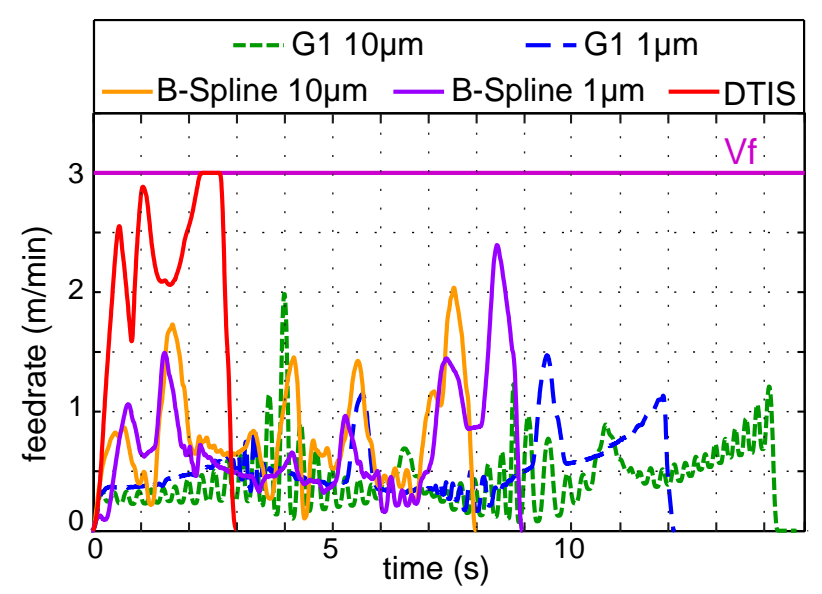

Fig. 7 Measured feedrate as a function of time for the 5 strategies

see further, this important variation of feedrate is harmful for the surface finish. On the second graph for G1 $1 \mu \mathrm{m}$, one can see that the feedrate is not linked to the NC block density. With native B-Spline strategies, the feedrate is everywhere higher than with the G1 strategies. However, low feedrate in dark blue is still present all along the tool path. Finally, with the DTIS method the feedrate is quite high all along the tool path without sharp oscillation. A feedrate reduction appears just before $Y=20 \mathrm{~mm}$ because of a rapid movement of the A axis caused by the rapid change of tool orientation. Fig. 8 shows that the feedrate is changing all along the tool path and sharp feedrate variations leave marks on the machined surface.

The reduction of the feedrate variations achieved by the DTIS method can also improve the cutting process as it leads to more constant cutting forces which can generate less geometrical errors caused by the deflection of the tool [13]. To go further in this direction, a feedrate scheduling which aims at maintaining constant cutting forces in 5-axis milling has been proposed in [11]. It is possible to go even further combining the jerk limited trajectory interpolation on the surface with process constraints such as in [7].

Fig. 9 presents on the left side the photo of the machined surface and the simulation of chordal deviations on the right side. The comparison between the photo of the final piece and the simulation of chordal deviation emphasizes the effect of the actual feedrate and slowdowns on surface finish and quality. The marks leaved by the feedrate oscillations are obvious when the surface is watched with different light orientations. Macro and micro-geometry measurements performed on a STIL micromeasure station equipped with an optical chromatic confocal point sensor confirm theses marks. It is difficult to give the same rendering with a static picture so the yellow wave signs emphasize the area where the main surface defects can be seen. 


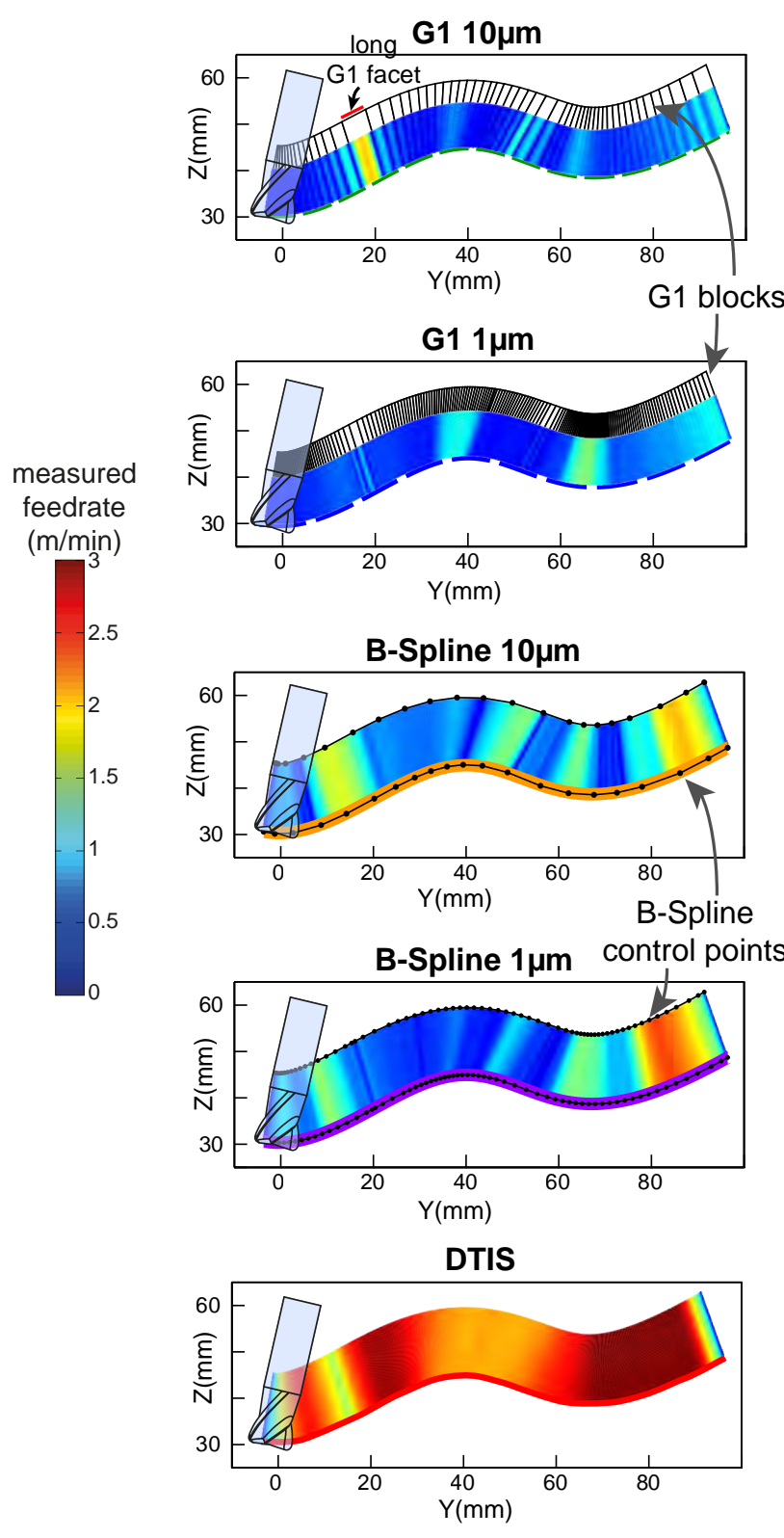

Fig. 8 Representation of the feedrate on the geometry for the 5 strategies

1. In the first portion of the surface machined with the $\mathrm{G} 1$ $10 \mu \mathrm{m}$ strategy, the long G1 facets are obvious on the photo. The sharp feedrate variations before and after these long facets leave important marks on the surface. The synchronization of the paths allows to repeat the mark in the $\mathrm{X}$ direction. At the end of this tool path strategy, there are no straight marks but the surface aspect is wavy. Simulation of chordal deviations confirms also high variations of geometry in this area.

2. With the second strategy, as there are fewer sharp feedrate variations and maximal chordal deviation is lower $(1 \mu \mathrm{m})$, the surface marks are reduced. Few waves still appear on the machined surface.
3. With the first native B-Spline strategy with $10 \mu \mathrm{m}$ tolerance, chordal deviations are similar to the G1 $10 \mu \mathrm{m}$ strategy, there are locally fewer oscillations. As feedrate is smoother, the oscillations of the feedrate still have an effect on the surface finish quality, but local marks caused by strong slowdowns are removed.

4. Reducing the tolerance from $10 \mu \mathrm{m}$ to $1 \mu \mathrm{m}$ with native B-Spline strategy reduces oscillations and thus surface marks, but the increase of control points reduces the average feedrate. Few waves can still be observed at the beginning of the paths.

5. Finally, the last portion of the machined surface proves experimentally the benefits of the DTIS method. There are no chordal deviations since the tool path is directly interpolated on the surface. Moreover, as the feedrate profile is smoother than with industrial strategies, the surface finish is better and no feed mark is left on the surface.

The application presented in this paper clearly shows the benefits of the Direct Trajectory Interpolation on the Surface in terms of productivity and surface quality. Thanks to the experimental validation on a 5-axis high speed machine and the comparison between the industrial strategies and the proposed method, it has been demonstrated that the machining time can be divided by more than two while improving the surface finish aspect.

\section{Conclusion}

Tool paths for free-form surface machining are usually approximated by CAM software. This approximation is induced by the limited format for geometry entities in the NC code. Indeed, the typical solution is to discretize smooth curves with small linear segments (G1) introducing geometrical deviations and tangency discontinuities. In the literature, exact tool path interpolation on the surface have been proposed but the kinematical constraints were not considered whereas the feedrate reductions are caused by the velocity, acceleration and jerk kinematical limitations. This paper proposes a Direct Trajectory Interpolation on the Surface with kinematical constraints in 5-axis. Once the new StepNC standard will be accepted by industrial CNC, this possibility to describe the tool path exactly will be useful for industrial applications. The experimental developments of the PREMIUM-OpenCNC allow to demonstrate experimentally the benefits of the proposed method. Indeed, the machining time reduction can be computed without machining experiments but the surface finish improvements cannot be assessed otherwise. Compared with G1 and native B-Spline interpolations, the DTIS interpolation can reduce the machining time from 2 to 4 times as well as improve the surface finish. As it is demonstrated in this paper, the amelioration of 

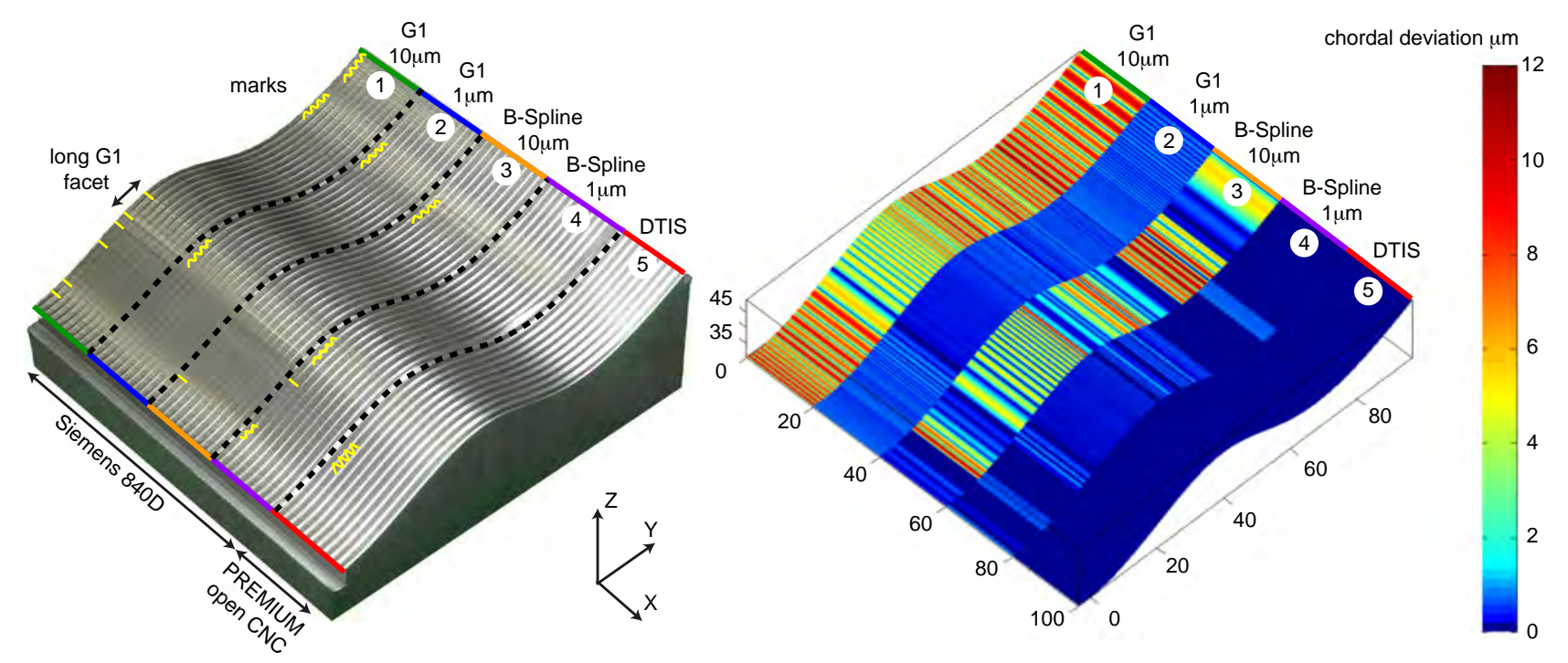

Fig. 9 Photo of machined free-form surface with feed marks and corresponding simulation of deviations

the data exchange between CAD/CAM and CNC will open new ways to optimize the manufacturing process.

Acknowledgements This work is supported by the French Ministry of Research and the Digiteo foundation (2010-47D).

\section{References}

1. (2014) LinuxCNC: Software for realtime control http://www.linuxcnc.org, accessed June 2014

2. Beudaert X, Lavernhe S, Tournier C (2012) Feedrate interpolation with axis jerk constraints on 5-axis NURBS and G1 tool path. Int J Mach Tool Manu 57(0):73 - 82, DOI 10.1016/j.ijmachtools.2012.02.005

3. Beudaert X, Lavernhe S, Tournier C (2013) 5-axis local corner rounding of linear tool path discontinuities. Int J Mach Tool Manu 73(0):9 - 16, DOI 10.1016/j.ijmachtools.2013.05.008

4. Boujelbene M, Moisan A, Tounsi N, Brenier B (2004) Productivity enhancement in dies and molds manufacturing by the use of $\mathrm{C} 1$ continuous tool path. Int J Mach Tool Manu 44(1):101 - 107, DOI 10.1016/j.ijmachtools.2003.08.005

5. Cheng CW, Tseng WP (2006) Design and implementation of a real-time NURBS surface interpolator. Int J Adv Manuf Tech 30(1-2):98-104, DOI 10.1007/s00170-005-0021-1

6. Erkorkmaz K, Yeung CH, Altintas Y (2006) Virtual CNC system - Part II - High speed contouring application. Int J Mach Tool Manu 46(10):1124 - 1138, DOI 10.1016/j.ijmachtools.2005.08.001

7. Erkorkmaz K, Layegh SE, Lazoglu I, Erdim H (2013) Feedrate optimization for freeform milling consider- ing constraints from the feed drive system and process mechanics. CIRP Annals - Manufacturing Technology 62(1):395 - 398, DOI 10.1016/j.cirp.2013.03.084

8. Farouki RT, Manjunathaiah J, Yuan GF (1999) G codes for the specification of Pythagorean-Hodograph tool paths and associated feedrate functions on openarchitecture CNC machines. Int $\mathbf{J}$ Mach Tool Manu 39(1):123 - 142, DOI 10.1016/S0890-6955(98)000182

9. ISO (2004) Industrial automation systems and integration - physical device control - data model for computerized numerical controllers - part 10: General process data. International Organization for Standardization ISO 14649-10

10. ISO (2004) Industrial automation systems and integration - physical device control - data model for computerized numerical controllers - part 11: Process data for milling. International Organization for Standardization ISO 14649-11

11. K SEL, Erdim H, Lazoglu I (2012) Offline force control and feedrate scheduling for complex free form surfaces in 5-axis milling. Procedia CIRP 1(0):96 - 101, DOI 10.1016/j.procir.2012.04.015

12. Koren Y, Lin RS (1995) Five-axis surface interpolators. CIRP Ann-Manuf Techn 44(1):379 - 382, DOI 10.1016/S0007-8506(07)62346-4

13. López de Lacalle L, Lamikiz A, Sánchez J, Salgado M (2004) Effects of tool deflection in the high-speed milling of inclined surfaces. Int $\mathbf{J}$ Adv Manuf Tech 24(9-10):621-631, DOI 10.1007/s00170-003-1723-x

14. Lartigue C, Thiebaut F, Maekawa T (2001) CNC tool path in terms of b-spline curves. Comput Aided Design 33(4):307 - 319, DOI 10.1016/S0010-4485(00)00090- 
7

15. Lartigue C, Tournier C, Ritou M, Dumur D (2004) High-performance NC for HSM by means of polynomial trajectories. CIRP Ann-Manuf Techn 53(1):317 320, DOI 10.1016/S0007-8506(07)60706-9

16. Liang H, Li X (2013) Five-axis STEP-NC controller for machining of surfaces. Int J Adv Manuf Tech 68(912):2791-2800, DOI 10.1007/s00170-013-4871-7

17. Lin RS (2000) Real-time surface interpolator for 3-D parametric surface machining on 3-axis machine tools. Int J Mach Tool Manu 40(10):1513 - 1526, DOI 10.1016/S0890-6955(00)00002-X

18. Lo CC (2000) CNC machine tool surface interpolator for ball-end milling of free-form surfaces. Int $\mathbf{J}$ Mach Tool Manu 40(3):307 - 326, DOI 10.1016/S08906955(99)00071-1

19. Omirou SL, Barouni AK (2005) Integration of new programming capabilities into a CNC milling system. Robot CIM-Int Manuf 21(6):518 - 527, DOI 10.1016/j.rcim.2004.10.002

20. Otsuki T, Ogino H, Ide S, Chiba T (2004) Curve interpolation method. Japan, Fanuc LTD. Yamanashi (US Patent 6823234 B2)

21. Pateloup V, Duc E, Ray P (2010) Bspline approximation of circle arc and straight line for pocket machining. Comput Aided Design 42(9):817 - 827, DOI 10.1016/j.cad.2010.05.003

22. Rauch M, Laguionie R, Hascoët JY, Suh SH (2012) An advanced STEP-NC controller for intelligent machining processes. Robot CIM-Int Manuf 28(3):375 - 384, DOI 10.1016/j.rcim.2011.11.001

23. Siemens (2009) Sinumerik - 5 axis machining. Doc order no 6FC5095-0AB10-0BP1

24. de Souza AF, Coelho R (2007) Experimental investigation of feed rate limitations on high speed milling aimed at industrial applications. Int J Adv Manuf Tech 32(1112):1104-1114, DOI 10.1007/s00170-006-0445-2

25. Tsai MC, Cheng CW, Cheng MY (2003) A real-time NURBS surface interpolator for precision three-axis CNC machining. Int J Mach Tool Manu 43(12):1217 - 1227, DOI 10.1016/S0890-6955(03)00154-8

26. Uhlmann E, Abackerli AJ, Schützer K, Lepikson HA, Helleno AL, Papa MCO, Conte EG, Mewis J (2014) Simulation and analysis of error impact on freeform surface milling. Int J Adv Manuf Technol 70(1-4):607620, DOI 10.1007/s00170-013-5280-7

27. Yang X (2002) Efficient circular arc interpolation based on active tolerance control. Comput Aided Design 34(13):1037 - 1046, DOI 10.1016/S00104485(01)00164-6

28. Yutkowitz SJ, Chester W (2005) Apparatus and method for smooth cornering in a motion control system. Siemens Energy \& Automation, Inc. Alpharetta, GA
(US Patent 6922606)

29. Zezhong CC, Maqsood KA (2014) A new approach to generating arc length parameterized NURBS tool paths for efficient three-axis machining of smooth, accurate sculptured surfaces. Int J Adv Manuf Tech 70(58):1355-1368, DOI 10.1007/s00170-013-5411-1 